\title{
Prognosis of the patients suffered from uterine carcinosarcoma from rural and urban areas
}

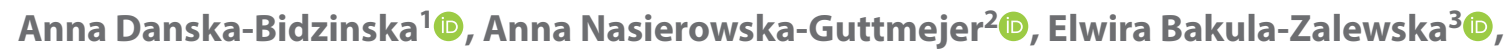 \\ Mariusz Bidzinski ${ }^{4} \mathbb{D}$, Waldemar Wierzba ${ }^{5}$ (D) \\ ${ }^{1}$ Chair and Department of Obstetrics, Gynaecology and Oncology, $2^{\text {nd }}$ Faculty of Medicine, Medical University of Warsaw, Poland \\ 2Department of Pathology, Central Clinical Hospital of the Ministry of Internal Affairs and Administration, Warsaw, Poland \\ ${ }^{3}$ Department of Pathology, Maria Sklodowska-Curie National Research Institute of Oncology, Warsaw, Poland \\ ${ }^{4}$ Gyneacologic Oncology Department, Maria Sklodowska-Curie National Research Institute of Oncology, Warsaw, Poland \\ ${ }^{5}$ University of Humanities and Economics in Lodz, UHE Satellite Campus in Warsaw, Poland
}

\begin{abstract}
Objectives: Uterine carcinosarcoma is a very aggressive neoplasm. Patients' median age at diagnosis ranges from 62 to 67 years. The aim of this study was to compare treatment results and prognostic factors for residents of urban and rural areas suffering from uterine carcinosarcoma.

Material and methods: Clinical outcomes of 58 uterine carcinosarcoma patients treated in one institution were assessed: 25 residents of rural and 33 of urban areas. All the patients were treated by using surgery followed by chemotherapy (48 pts) or radiotherapy (10 pts). Standard chemotherapy regimen comprised of paclitaxel $175 \mathrm{mg} / \mathrm{m}^{2}$ and carboplatin on day one at area under curve (AUC) six every 21 days. Radiotherapy was performed by combined treatment - tele and brachytherapy. External beam pelvic radiation therapy (EBRT) once a day, five days a week with a daily fraction size of $1.8 \mathrm{~Gy}$ over five weeks at cumulative dose 50.4 Gy was the first part of adjuvant treatment. High-dose-rate (HDR) brachytherapy at dose 22.5 Gy was the second part of radiotherapy.

Results: A strong correlation between tumor diameter and the presence of lymph node metastasis was observed. Tumor size greater then $4.5 \mathrm{~cm}$ correlated with presence of node involvement and this parameter was statistically significant $(p=0.015)$. There was no significant correlation between other analyzed clinical factors and overall survival. In the period 2004-2010 43.5\% (10/23) and 50\% (14/28) of rural and urban residents, respectively, died due to carcinosarcoma progression.

Conclusions: Uterine carcinosarcoma patients in rural and urban areas seem to have similar outcomes.

Key words: carcinosarcoma; rural area; urban area; overall survival; overall survival
\end{abstract}

Ginekologia Polska 2021; 92, 11: 774-777

\section{INTRODUCTION}

Uterine carcinosarcomas are rare tumors. It represents less than five percent of all uterine malignant tumors. In the US, the incidence of carcinosarcoma is approximately 1 to 4 per 100000 women [1]. There are no detailed Polish dates about incidence of carcinosarcoma. Carcinosarcomas are recognized in women with the median age at diagnosis ranging from 62 to 67 years. Incidence of uterine carcinosarcoma among blacks is a twofold higher compared with non-Hispanic whites [2]. Uterine carcinosarcomas risk factors are similar to endometrial carcinomas. Both malignancies are associated with nulliparity, use of exogenous estrogen and tamoxifen and obesity [3]. Progestin-containing contra- ceptives are protective against both types of neoplasms. Previous exposure to pelvic radiation can also increase risk of developing uterine carcinosarcoma [4].

There is no exact information concerning the incidence of uterine carcinosarcoma in rural and urban areas. In some South America countries, there are reports presenting distribution of gynecological cancers in these two areas $[5,6]$. Epidemiology registries have shown that the incidence of uterine cancer in rural regions is lower than in urban area. It has been established that overall incidence and mortality rates of malignant neoplasms are lower among rural citizen in comparison with urban residents $[7,8]$. This difference in cancer occurrence may be explained in part to personal

\footnotetext{
Corresponding author:

Waldemar Wierzba

University of Humanities and Economics in Łódź, UHE Satellite Campus in Warsaw, Poland

e-mail:wwierzba@post.pl
} 
health habits such as cigarette and alcohol consumption, overweight in urban citizens [8]. Differential distributions of environmental risk factors should be also important factors influencing on this epidemiological issue.

The aim of this study was analysis the survival rates and some prognostic factors of uterine carcinosarcoma among residents of rural and urban areas in Poland.

\section{MATERIAL AND METHODS}

Clinical outcomes of 58 uterine carcinosarcoma patients treated at the Maria Sklodowska-Curie National Institute of Oncology in Warsaw between 2004-2011 were analyzed. There were 25 rural and urban 33 residents among uterine carcinosarcoma patients. Histopathological diagnosis was assessed independently by two pathologists (AN-G and EB-Z) for all the tumors. The average age of the analyzed group of patients was 62 years (47-78 range).

All women were treated by combined methods. After surgery in 48 patients, chemotherapy was given and in an additional 10 cases, radiotherapy was performed. In 15 women a simple hysterectomy with bilateral salpingoophorectomy was performed. In the remaining group of patients, radical hysterectomy, pelvic and periaortic lymphadenectomy were done. Standard chemotherapy regimen consisted of paclitaxel $175 \mathrm{mg} / \mathrm{m}^{2}$ and carboplatin on day one at area under curve (AUC) 6 every 21 days. Most of the patients (46) received six cycles of chemotherapy while two women were withdrawn from chemotherapy after four cycles because of its toxicity. Ten patients received external beam pelvic radiation therapy (EBRT) once a day, five days a week in 26 fractions at cumulative dose $50.4 \mathrm{~Gy}$ and high-dose-rate (HDR) brachytherapy at dose $22.5 \mathrm{~Gy}$.

Prognosis analyses were performed taking into consideration the following clinical aspects: age, clinical stage, size of the tumor, time from diagnosis to surgery, time of adjuvant treatment and lymph node status. All the examined parameters were stratified according the rural or urban patients' residency. No patients were lost and the mean time of follow up was 247 weeks (149-482 weeks range).

Statistical analysis was performed using STATISTICA software (StatSoft). Chi2 and Pearson test was used for testing sample variance. Log rank test was used for comparing survival distributions in the analyzed groups of patients. Kaplan-Meier curves were generated for visualization of survival data.

\section{RESULTS}

FIGO stage of uterine carcinosarcoma of patients of rural or urban residency is presented in Table 1. There were no statistical differences in clinical stage distribution between the two groups of patients. Tumor size ranged from 1 to
$6 \mathrm{~cm}$ (mean $3.5 \mathrm{~cm}$ ). No difference was observed in tumor diameter among rural and urban residents.

Mean period from diagnosis to treatment was 4.2 weeks (range 3-7 weeks). Mean time of postoperative chemotherapy was 18.6 weeks (range 16-21 weeks). Adjuvant radiotherapy lasted on average 6.1 weeks (range 5-7 weeks). There were no statistically significant differences in factors mentioned above between either analyzed group. Eleven out of 23 rural residents (44\%) and 16 out of 33 women from the urban subgroup (48.5\%) died. The mean time to progression was 68 and 85 weeks for urban and rural residents, respectively and it was not statistically significant. The mean time to death was 149 and 183 weeks for urban and rural groups; again, the difference was not statistically significant. Two patients from the rural area died due to no oncological reason. One died because of cardiac infarction and the second reason was cerebral stroke. Some of the variables in both subgroups of uterine carcinosarcoma patients are presented in Table 2. A strong correlation in Pearson test was observed between tumor diameter and lymph node involvement. Tumor size greater than $4.5 \mathrm{~cm}$ was found to correlate with presence of node metastases $(p=0.015)$.

Next, we analyzed the correlation between the presence of lymph node metastases and uterine carcinosarcoma patients' overall survival (Fig. 1) Chi2 $=15.015$, $\mathrm{df}=2 \mathrm{p}<0.015$. Figure 2 and 3 present Kaplan-Meier curves

\begin{tabular}{|l|l|l|l|}
\hline \multicolumn{5}{|l|}{ Table 1. Clinical stage in analyzed group } \\
\hline FIGO Stage & Rural residents & Urban residents & p \\
\hline la & 3 & 3 & \\
\hline Ib & 12 & 14 & \\
\hline II & 5 & 8 & NS \\
\hline IIlla & 1 & 0 & \\
\hline IIIb & 1 & 1 & \\
\hline IIIC & 3 & 7 & \\
\hline All & 25 & 33 & \\
\hline
\end{tabular}

Table 2. Analysis of some clinical variable stratified according to site of living

\begin{tabular}{|l|l|l|}
\hline Variable & $\mathbf{X}^{\mathbf{2}}$ value & $\mathbf{p}$ \\
\hline Clinical stage & 7.421 & 0.191 \\
\hline Tumor size & 4.447 & 0.487 \\
\hline Age & 9.125 & 0.104 \\
\hline Time to beginning of treatment & 8.705 & 0.069 \\
\hline Treatment time & 10.393 & 0.238 \\
\hline Radiotherapy & 3.389 & 0.142 \\
\hline Chemotherapy & 4.075 & 0.396 \\
\hline Number of recurrences & 1.369 & 0.241 \\
\hline Number of die (deaths) & 1.062 & 0.957 \\
\hline
\end{tabular}




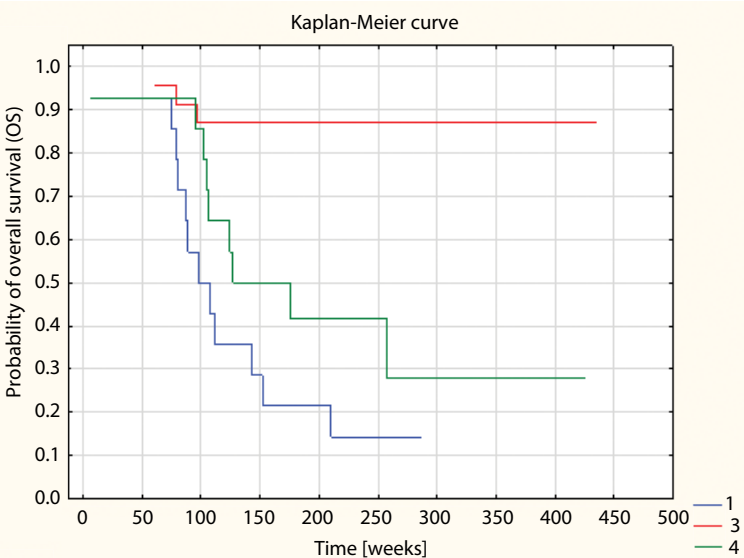

Figure 1. The impact of nodal status on overall survival of carcinosarcoma patients; 1 -lymph node metastasis; 3 - without lymph node metastasis; 4-unknown nodal status; $\mathrm{Chi}^{2}=15.015$; $\mathrm{df}=2 ; \mathrm{p}=0.00055$

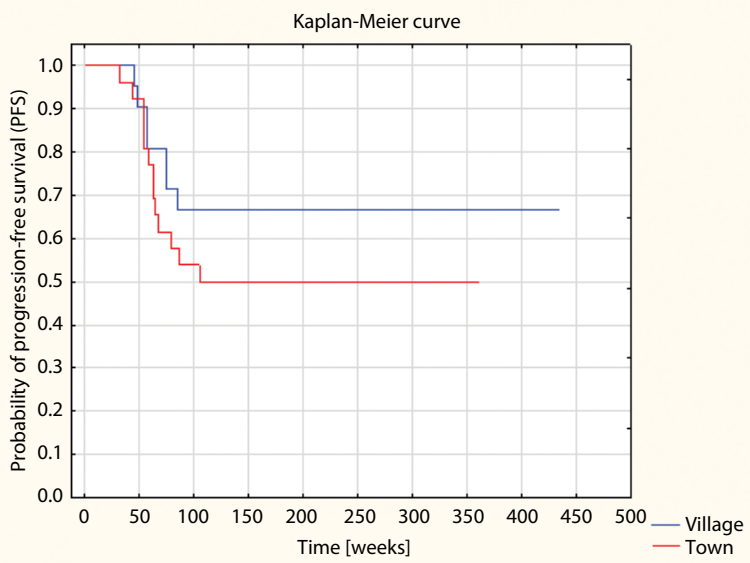

Figure 2. Progression free survival among uterine carcinoma patients of rural and urban residency

for progression free survival (log-rank test $p=0.265$ ) and overall survival (log-rank test $p=0.209$ ) of uterine carcinosarcoma patients, respectively, according to their residency. There was no significant difference in terms of survival between the two analyzed groups of patients.

\section{DISCUSSION}

Carcinosarcoma are mixed tumors with both mesenchymal and epithelial components of malignant nature. These tumors are considered as one of the most aggressive uterine neoplasms. Compared to endometrial carcinoma, carcinosarcoma develops in older age groups. Risk factors of these malignancies are similar. Pelvic irradiation, exposure to exogenous estrogens or tamoxifen administration are the most common risk factors [8].

Differences in survival among rural and urban residents were observed in certain types of malignancies $[8,9]$. Resi-

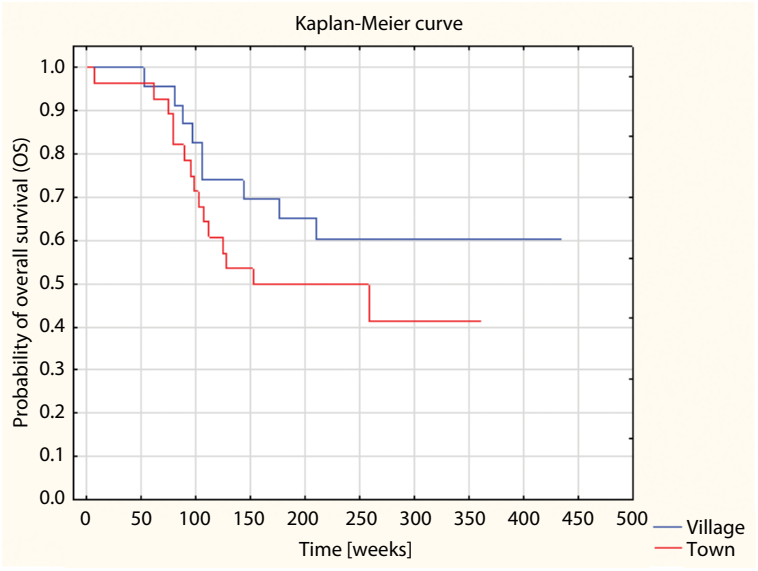

Figure 3. Overall survival among uterine carcinoma patients of rural and urban residency

dents of the rural areas more often have unstaged cancers in comparison with urban residents. Among the women with known stages at diagnosis, rural residents usually have more advanced disease than urban patients. Taking into consideration the thesis mentioned above, our analysis should show if the same patterns have been observed in carcinosarcoma patient's.

Our analysis has shown the same clinical characteristics in both groups of residents. There were no differences in the clinical stage, time of therapy or the entire treatment duration. The incidence of carcinosarcoma was comparable both in rural and urban areas. The similar results have been presented in ovarian cancer patients by Szpurek et al. [10]. There are different epidemiological data in cases of endometrial and cervical cancer. The Polish National Registry has shown less incidents of cervical and endometrial cancers in rural areas than towns and cities.

We did not observe any discrepancy in staging or diagnostic procedures between rural and urban residents. It should be stressed that the time from diagnosis of disease to surgery lasted on average 4.2 weeks. This time was comparable in both group of residents. Unfortunately, we have no exact data of time duration from beginning of symptoms to diagnosis of malignancy.

The comparison of progression free survival (PFS) and overall survival (OS) does not allow to achieve statistical significance although a tendency of higher values in rural residents was observed. This fact may be explained by a healthier environment in rural areas as well as less hormonal consumption by rural women [11-14].

In most of patients, within the analyzed group, early clinical stages have been diagnosed. FIGO stage I and II included 45 out of 58 patients. Medium five-years survival for the total population was $53 \%$, which is compatible with other observations. Bosquet et al. [12], analyzed 121 patients 
with carcinosarcoma treated with adjuvant chemotherapy following surgery. In this study the five-year survival for stage I and II was 59\%, for stage III was 22\% and for stage IV 9\% [15].

In the analyzed group of patients, chemotherapy and radiotherapy were used as adjuvant treatment, but chemotherapy was given to most of the patients. The role of postoperative irradiation was assessed in several retrospective series but in only few randomized trials. The Gynecologic Oncology Group compared whole abdomen - pelvic irradiation to three cycles of chemotherapy based on cisplatin and ifosfamide as adjuvant therapy after surgery, in 206 eligible patients. The estimated death rate was $29 \%$ lower with chemotherapy when compared to radiation therapy. This trial has given rise to future clinical trials evaluating adjuvant chemotherapy, which has been accepted as standard treatment nowadays [16-18]. The combination of carboplatin and paclitaxel replaced doublet regimen mentioned above due to less toxicity [19]. It is also our standard regimen which was practiced in analyzed population. Radiotherapy was used as adjuvant treatment in the past, but since 2008 , this method is practiced mostly in recurrences [20].

Nodal status appeared to be the most significant prognostic factor in carcinosarcoma. Therefore, pelvic and paraaortic lymphadenectomy is mandatory in staging procedures. Regarding its impact on survival, the most of studies confirm a significant survival benefit resulting from lymphadenectomy [21]. The possible explanation of survival improvement associated with lymphadenectomy may include removal of micro-metastatic foci within lymph nodes. The role of enlarged lymph nodes removal is no doubt. Our study also confirms the influence of lymphadenectomy on overall survival.

\section{CONCLUSIONS}

Retrospective analysis made a bias on the final conclusions of this article, but a prospective trial would be difficult to conduct in such rare tumors. It should be stressed that residents of rural and urban areas have similar prognosis in this type of malignancy. No statistically significant differences in overall survival, progression-free survival and proportional distribution of clinical stage uterine carcinosarcoma between rural and urban residents may be cause by available and well-functioning health system in these areas.

\section{Conflict of interest}

There is no conflict of interest by the authors.

\section{REFERENCES}

1. Cantrell LA, Blank SV, Duska LR. Uterine carcinosarcoma: A review of the literature. Gynecol Oncol. 2015; 137(3): 581-588, doi: 10.1016/j. ygyno.2015.03.041, indexed in Pubmed: 25805398.

2. Hosh M, Antar S, Nazzal A, et al. Uterine Sarcoma: Analysis of 13,089 Cases Based on Surveillance, Epidemiology, and End Re- sults Database. Int J Gynecol Cancer. 2016; 26(6): 1098-1104, doi: 10.1097/IGC.0000000000000720, indexed in Pubmed: 27177280.

3. Denschlag D, Ulrich UA. Uterine uarcinosarcomas - diagnosis and management. Oncol Res Treat. 2018; 41(11): 675-679, doi: 10.1159/000494335, indexed in Pubmed: 30317231.

4. Cherniack AD, Shen $\mathrm{H}$, Walter V, et al. Cancer Genome Atlas Research Network. Integrated molecular characterization of uterine carcinosarcoma. Cancer Cell. 2017; 31(3):411-423, doi: 10.1016/j.ccell.2017.02.010, indexed in Pubmed: 28292439.

5. Dey S, Hablas A, Seifeldin IA, et al. Urban-rural differences of gynaecological malignancies in Egypt (1999-2002). BJOG: An International Journal of Obstetrics \& Gynaecology. 2009; 117(3): 348-355, doi: 10.1111/j.14 71-0528.2009.02447.x.

6. Alshahrani S, Hablas A, Chamberlain RM, et al. Changing Incidence of Uterine Cancer in Rural Egypt: Possible Impact of Nutritional and Epidemiologic Transitions. J Glob Oncol. 2019; 5: 1-7, doi: 10.1200/JGO.18.00255, indexed in Pubmed: 31365301.

7. Garg G, Yee C, Schwartz K, et al. Patterns of care, predictors, and outcomes of chemotherapy in elderly women with early-stage uterine carcinosarcoma: a population-based analysis. Gynecol Oncol. 2014; 133(2):242-249, doi: 10.1016/j.ygyno.2014.02.021, indexed in Pubmed: 24561247.

8. Park J, Blackburn BE, Rowe K, et al. Rural-metropolitan disparities in ovarian cancer survival: a statewide population-based study. Ann Epidemiol. 2018; 28(6): 377-384, doi: 10.1016/j.annepidem.2018.03.019, indexed in Pubmed: 29705053.

9. Singh R. Review literature on uterine carcinosarcoma. J Cancer Res Ther. 2014; 10(3): 461-468, doi: 10.4103/0973-1482.138197, indexed in Pubmed: 25313723.

10. Szpurek D, Moszynski R, Szubert S, et al. Urban and rural differences in characteristics of ovarian cancer patients. Ann Agri Environ Med. 2013; 20(2): 390-94.

11. Zahnd WE, James AS, Jenkins WD, et al. Rural-urban differences in cancer incidence and trends in the United States. Cancer Epidemiol Biomarkers Prev. 2018; 27(11): 1265-1274, doi: 10.1158/1055-9965.EPI-17-0430, indexed in Pubmed: 28751476.

12. Strosnider $\mathrm{H}$, Kennedy $\mathrm{C}$, Monti $\mathrm{M}$, et al. Rural and urban differences in air quality, 2008-2012, and Community Drinking Water Quality, 20102015 - United States. MMWR Surveill Summ. 2017; 66(13): 1-10, doi: 10.15585/mmwr.ss6613a1, indexed in Pubmed: 28640797.

13. Stamenić V, Strnad M. Urban-rural differences in a population-based breast cancer screening program in Croatia. Croat Med J. 2011; 52(1): 76-86, doi: 10.3325/cmj.2011.52.76, indexed in Pubmed: 21328724.

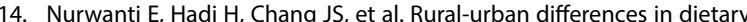
behavior and obesity: results of the riskesdas study in 10-18-year-old Indonesian children and adolescents. Nutrients. 2019; 11(11), doi: 10.3390/nu11112813, indexed in Pubmed: 31752101.

15. Gonzalez Bosquet J, Terstriep SA, Cliby WA, et al. The impact of multi-modal therapy on survival for uterine carcinosarcomas. Gynecol Oncol. 2010; 116(3):419-423, doi: 10.1016/j.ygyno.2009.10.053, indexed in Pubmed: 19896181

16. Gokce ZK, Turan T, Karalok A, et al. Clinical outcomes of uterine carcinosarcoma: results of 94 patients. Int J Gynecol Cancer. 2015; 25(2): 279-287, doi: 10.1097/IGC.0000000000000347, indexed in Pubmed: 25611900.

17. Wolfson $A H$, Brady MF, Rocereto T, et al. A gynecologic oncology group randomized phase III trial of whole abdominal irradiation (WAI) vs. cisplatin-ifosfamide and mesna (CIM) as post-surgical therapy in stage I-IV carcinosarcoma (CS) of the uterus. Gynecol Oncol. 2007; 107(2): 177-185, doi: 10.1016/j.ygyno.2007.07.070, indexed in Pubmed: 17822748.

18. Magnuson WJ, Petereit DG, Anderson BM, et al. Impact of adjuvant pelvic radiotherapy in stage I uterine sarcoma. Anticancer Res. 2015; 35(1): 365-370, indexed in Pubmed: 25550573.

19. Powell MA, Filiaci VL, Rose PG, et al. Phase Il evaluation of paclitaxel and carboplatin in the treatment of carcinosarcoma of the uterus: a Gynecologic Oncology Group study. J Clin Oncol. 2010; 28(16): 2727-2731, doi: 10.1200/JCO.2009.26.8326, indexed in Pubmed: 20421537.

20. Galaal K, van der Heijden E, Godfrey K, et al. Adjuvant radiotherapy and/or chemotherapy after surgery for uterine carcinosarcoma. Cochrane Database Syst Rev. 2013(2): CD006812, doi: 10.1002/14651858. CD006812.pub3, indexed in Pubmed: 23450572.

21. Seagle BLL, Kanis M, Kocherginsky M, et al. Stage I uterine carcinosarcoma: Matched cohort analyses for lymphadenectomy, chemotherapy, and brachytherapy. Gynecol Oncol. 2017; 145(1): 71-77, doi: 10.1016/j. ygyno.2017.01.010, indexed in Pubmed: 28317560 